\title{
Incobotulinumtoxin Diluted in Zinc Gluconate Solution for Facial Wrinkles: Randomized Clinical Trial
}

\author{
Leonardo Oliveira Ferreira Bianquini ${ }^{1,2 *}$, Paula Força Dellacqua ${ }^{2}$, Lúcia Helena Sagrillo Pimassoni ${ }^{3}$, and Denise Cantarelli \\ Machado Machado ${ }^{4}$ \\ ${ }^{1}$ Coordinator of the Health Sciences Research Institute (FAPECS) Serra, ES, Brazil \\ ${ }^{2}$ Dermatology Department, Health Sciences Research Institute (FAPECS) Serra, ES, Brazil \\ ${ }^{3}$ School of Science, Santa Casa de Misericórdia de Vitória (EMESCAM), Vitória, Espirito Santo, Brazil \\ ${ }^{4}$ School of Medicine, Coordinator of Preclinical Research Center of Brain Institute at Pontifical Catholic University of Rio Grande do Sul (PUCRS) \\ Porto Alegre, Rio Grande do Sul, Brazil
}

*Corresponding author: Leonardo Oliveira Ferreira Bianquini, Instituto de Pesquisa em Ciências da Saúde (FAPECS), n0 300, Torre A, sala 410, CEP 29160161, Serra, Espírito Santo, Brasil; E-mail: leonardobianquini@yahoo.com

Received: February 24, 2021; Accepted: March 01, 2021; Published: March 05, 2021

\begin{abstract}
Background: We describe the results of the application of Incobotulinumtoxin A reconstituted in a solution composed of zinc gluconate $0.02 \%$ and we evaluated whether the presence of zinc would increase or not the duration of treatment.

Methods: The sample consisted of 48 female participants, over 50 years old, with no history of chronic gastrointestinal disease or diabetes, and who had not undergone treatment with botulinum toxin less than 6 months ago. All patients answered the questionnaire with socio-demographic and health questions in which the patient's perception of her quality of life was assessed through validated WHOQOL-Bref questionnaire before and after application.

Results: The results obtained showed that there is no relationship between race, alcoholism, smoking and use of sunscreen with the effect of the toxin in the two study groups. Concerning the variables age and amount of weekly zinc intake, the result of the Manny-Whitney test indicated that there is no significant difference between the groups, $\mathrm{p}$-value $>0.05$. All patients were evaluated with the frontalis muscle at rest and in movement. In the group of patients who received solubilized incobotulinumtoxine A in sodium chloride, within 14 weeks of application there was a $66 \%$ reduction in the effects in patients with frontal wrinkles at rest. In the group of patients who received incobotuliniumtoxin A solubilized in zinc gluconate, within 14 weeks of application, there was a 100\% reduction in effects in patients with frontal wrinkles at rest.
\end{abstract}

Conclusions: The data identified that the patients' lifestyle habits do not influence the final result of the procedure, and that the duration of the effect is not linked to the dilution of the product with a substance different from the classic dilution with physiological saline solution.

Keywords: Zinc, Botulinum toxins type A, Facial muscles, Efficiency, Side effects, Adverse drug-related reactions, Record of clinical trial: ISRCTN27486491

\section{Introduction}

Botulinumtoxin type A is a zinc-dependent endoprotease that acts on vulnerable cells to separate the polypeptides, which are essential for the cation. When the exogenous $\mathrm{Zinc}\left(\mathrm{Zn}^{2+}\right)$ cation is added to the toxin that was removed by soluble chelators, the molecule coats the cation and recovers the activity of catalytic and neuromuscular block. Exogenous $\mathrm{Zn}^{2+}$ can restore activity of the toxin, either when the toxin is free in solution outside the cell or when internalized in cytosol [1]. Because they are zinc-dependent proteases, the question arose whether the intracellular zinc concentration could influence the action of the toxin [2].

\section{Materials and Methods}

This is a prospective, double-blind study. The sample size was composed of 48 individuals and was calculated by use of the G-Power program to verify the association between variables. For this purpose, an effect of 0.5 was used, a test power of $80 \%$ and a confidence level of $95 \%$. The sample was composed of female participants, over 50 years of age, without a history of chronic gastrointestinal disease or diabetes, and who had not undergone treatment with botulinum toxin less than 6 months ago. Every patient answered the questionnaire with sociodemographic and health questions, in which the patient's perception of her quality of life was assessed through the validated WHOQOLbref ${ }^{11}$ questionnaire before and after application. The study assessed the effectiveness and duration of the effect of botulinumtoxin type A (Incobotulinium) reconstituted in a $0.02 \%$ zinc gluconate solution versus dilution in $0.9 \%$ saline. The participants were photographed in similar lighting conditions before treatment in weeks $0,2,4$ and 14 after the procedure. 


\section{Discussion}

The results obtained showed that there is no relationship between race, alcoholism, smoking and use of sunscreen with the effect of the toxin in the two study groups. Concerning the variables age and amount of weekly zinc intake, the result of the Manny-Whitney test showed that there is no significant difference between the groups, $\mathrm{p}$-value> 0.05 . Before checking the duration of the relaxing effect of the frontalis muscle in the application of botulinum toxin solubilized with $0.9 \%$ sodium chloride and with zinc gluconate $0.02 \%$, the individualized views of patients and the examining physician regarding the degree of front line wrinkles [3] that each participant presented before the treatment. The result indicated that there was agreement between the patients and the doctor, that is, when the participant looked at herself in the mirror and compared it to the scale of the lines the doctor also found similar results. Another study [4] which also used the same scale of front lines to check the duration of three types of botulinum toxin type A in 180 patients (incobotulinumtoxin A, onabotulinumtoxin A, and abobotulinumtoxin A) considered only the opinion of subjects and did not compare the doctor's point of view. The beginning of the treatment was defined as the fourteenth day (or second week) after execution of the application, since the effect of botulinum toxin, according to the literature [5] is complete in two weeks, enough time for the observer and patient to find a decrease in frontal muscle activity compared to photographs of two weeks before treatment. The calculation also included the twenty-eighth day and finally the ninety-eighth day (or 14th weeks) after application. All patients were assessed with the frontalis muscle at rest and in motion. In the group of patients who received incobotulinumtoxin A solubilized in sodium chloride, within 14 weeks of application there was a $66 \%$ reduction of effects in patients with frontal wrinkles at rest. On the other hand, the patients who were asked to move the frontal muscle (movement) there was a reduction of $75 \%$ of the desired effect within 98 days of application. Moving wrinkles seem to tend to return to the initial moment earlier compared to the resting wrinkles. The results of this study are consistent with previous6 research that demonstrated that intramuscular treatment with botulinum toxin type improves the appearance of lines at rest and overall skin quality. One possible explanation for this effect is that muscle weakness eliminates the fold the repetitive fold of the skin and relieves chronic stress applied to overlapping skin, which causes elastin and collagen to be strengthened in these places over time [6]. In the group of patients who received incobotuliniumtoxin A solubilized in gluconate zinc, after 14 weeks of application there was a $100 \%$ reduction in the effects on patients with frontal wrinkles at rest. The patients who were asked to move the frontalis muscle there was also a $75 \%$ reduction in the effect within 98 days of application, corroborating with a study [7] that compared two presentations of Botulinum Toxin Type A (onabotulinium toxin A and incobotulinium toxin A). There are many myths that involve how to handle the botulinum toxin, many doctors use it only if they are reconstituted on the day of application, however, a study [8] confirmed that Botulinum Toxin Type A is an extremely stable molecule, and vigorous stirring does not impair its potency, even after 6 weeks of dilution. The results of the present study also showed that individuals who consume more zinc-rich foods, regardless of whether they were treated with incobotulinumtoxin A diluted in chloride sodium or zinc gluconate, had no more lasting effect. This variant was not evaluated by the author who described the oral use of zinc to increase the duration of effects of botulinum toxin [2]. This study evaluated whether some factors such as alcoholism, smoking, use of sunscreen and race could influence the results, considering the frontal wrinkles at rest and movement. It is known that wrinkles are also associated with aging, hormonal status, smoking and illness complications [9] and that there is a link between perioral wrinkles and smoking and that smoking is a risk factor for premature skin aging. Studies have proposed that cigarette exposure increases matrix- 1 and 3 metalloproteinase activity and decreases the production of pro-collagen in the skin, possibly due to the production of free radicals induced by tobacco $[10,11]$. However, this study has not demonstrated therapeutic success or shorter duration in current smoking patients who received botulinum toxin applications with both dilutions.

\section{Conclusions}

The data identified that the duration of the effect of incobotulinumtoxin A diluted with Zinc gluconate was not superior to dilution with $0.9 \%$ sodium chloride. However, the study demonstrated that in patients who drink alcohol and who used botulinum toxin diluted in zinc gluconate $0.02 \%$ there seems to be a better efficiency. Another finding was that the habit of not using sunscreen does not influence the effect of Botulinum Toxin duration. Patients with low or high phototypes have the same duration of effect as botulinum toxin.

\section{Acknowledgments}

We thank all patients who participated in this study.

\section{Financial Disclosure}

None to declare.

\section{Conflict of Interest}

All cited authors declare that they have no conflict of interest to disclose.

\section{Informed Consent}

All participants agreed to the survey and signed a free and informed consent form.

\section{Author Contributions}

Leonardo Bianquini wrote the manuscript; Denise Cantarelli approved the final version; Paula Dellacqua collected data for review; Lúcia Pimassoni did a statistical analysis.

\section{Data Availability}

The data supporting the findings of this study are available from the corresponding author upon reasonable request.

\section{References}

1. SIMPSON LL, et al. (2001) The role of zinc binding in the biological activity of botulinum toxin. J Biol Chem 20(29): 27034-41. [crossref]

2. KOSHY JC, et al. (2012) Effect of dietary zinc and phytase supplementation on botulinum toxin treatments. Journal of Drugs in Dermatology 11(4): 507-512. [crossref] 
3. MERZ AESTHETICS. (2016) Upper face, Disponível. Acesso em: [crossref]

4. RAPPL T, et al. (2013) Onset and duration of effect of incobotulinumtoxinA, onabotulinumtoxinA, and abobotulinumtoxinA in the treatment of glabellar frown lines: a randomized, double-blind study. Clinical, Cosmetic and Investigational Dermatology 24(6): 211-9. [crossref]

5. CHEN JJ; DASHTIPOUR, K. Mar. (2013) Abo-, inco-, ona-, and rima-botulinum toxins in clinical therapy: a primer. Pharmacotherapy 33(3): 304-18. [crossref]

6. CARRUTHERS, A. Sep. (2016) Repeated OnabotulinumtoxinA treatment of glabellar lines at rest over three treatment cycles. Dermatol Surg 42(9): 1094-101. [crossref]
7. INUI K (2012) Toxic and nontoxic components of botulinum neurotoxin complex are evolved from a common ancestral zinc protein. Biochem Biophys Res Commun 419(3): 500-4. [crossref]

8. SHOME D, et al. (2010) Botulinum toxin A: is it really that fragile a molecule? Dermatologic Surgery 36(4): 2106-2110. [crossref]

9. MANRÍQUEZ JJ, et al. (2014) Wrinkles. BMJ Clin Evid. [crossref]

10. CHIEN AL, et al. (2016) Perioral wrinkles are associated with female gender, aging, and smoking: development of a gender-specific photonumeric scale. Journal of the American Academy of Dermatology, 74(5): 924-930. [crossref]

11. Development of the World Health Organization WHOQOL-BREF quality of life assessment. The WHOQOL Group. Psychol Med 1998 28(3): 551-8. [crossref] 